\title{
Theoretical progress in describing the $B$-meson lifetimes
}

\section{DAMIR BECIREVIC*}

Dipartimento di Fisica, Università di Roma "La Sapienza",

Piazzale Aldo Moro 2, I-00185 Roma, Italy

E-mail: 'Damir. Becirevicoroma1.infn.ití

ABSTRACT: The present status of the theoretical estimates of the difference between the widths of the neutral $B_{s}$-mesons and of the $B$-meson lifetime ratios is reviewed. In particular, the lattice results for the matrix elements of the relevant $\Delta B=2$ operators are updated and the first lattice QCD results for the matrix elements of $\Delta B=0$ operators are presented. In both cases, the NLO perturbative QCD corrections in the coefficient functions have been included. The theoretically updated results are: $(\Delta \Gamma / \Gamma)_{B_{s}}=7 \pm 4 \%$, $\tau\left(B^{+}\right) / \tau\left(B_{d}\right)=1.07(3)$ and $\tau\left(B_{s}\right) / \tau\left(B_{d}\right)=1.00(2)$.

I discuss the following two topics:

$\circ(\Delta \Gamma / \Gamma)_{B_{s}}$, the quantity that recently attracted quite a bit of attention among theorists and for which the experimental upper limit has been set at [i]i]:

$$
\left(\frac{\Delta \Gamma}{\Gamma}\right)_{B_{s}}<0.31 \quad(95 \% \text { C.L. })
$$

$\circ \tau\left(B_{u(s)}\right) / \tau\left(B_{d}\right)$ have been measured quite accurately [i,

$$
\frac{\tau\left(B_{u}\right)}{\tau\left(B_{d}\right)}=1.07(2), \quad \frac{\tau\left(B_{s}\right)}{\tau\left(B_{d}\right)}=0.95(4) .
$$

Important theoretical progress in computing these ratios has been made this year. I will not discuss the theoretical predictions for the ratio $\tau\left(\Lambda_{b}\right) / \tau\left(B_{d}\right)$, where, in my opinion, substantial progress is yet to be made.

Theoretical set-up for both of the above topics relies on the hypothesis of the (global and local) quark-hadron duality [2]. The validity of that assumption is not totally clear, although the impressive agreement of many theoretical predictions in $\tau$-physics (for which the duality has been assumed) with the precise experimental data is very encouraging [3]

${ }^{*}$ Speaker. 


\section{WIDTH DIFFERENCE OF THE $B_{S}^{0}$-SYSTEM}

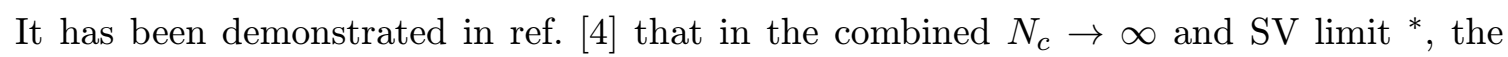
quark-hadron duality for $\Delta \Gamma_{B_{s}}$ indeed works. Out of that limit, however, the quark-hadron duality is again an assumption.

The (modern) theoretical expression, based on the operator product expansion (OPE),

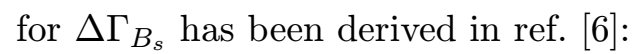

$$
\Delta \Gamma_{B_{s}}=\frac{G_{F}^{2} m_{b}^{2}}{12 \pi m_{B_{s}}}\left|V_{c b}^{*} V_{c s}\right|^{2}\left\{G_{1}(\mu)\left\langle\bar{B}_{s}\left|O_{1}(\mu)\right| B_{s}\right\rangle+G_{2}(\mu)\left\langle\bar{B}_{s}\left|O_{2}(\mu)\right| B_{s}\right\rangle+\delta_{1 / m_{b}}\right\},
$$

where the flavour structure of the operators $O_{1,2}(\mu)$ is $\Delta B=2 ; \delta_{1 / m_{b}}$ contains all the contributions from the $1 / m_{b}$ corrections to the first two terms. Corrections $\propto 1 / m_{b}^{n \geq 2}$ are neglected.

2. Short distance physics is encoded in the functions $G_{1,2}(\mu)$ which are the combinations of $\Delta B=1$ Wilson coefficients. The next-to-leading order (NLO) corrections to these functions have been computed in ref. [i] $]$, where the authors also kept the ratio $m_{c} / m_{b} \neq 0$. The residual scale dependence of $\Delta B=1$ Wilson coefficients entering the functions $G_{1,2}\left(m_{b}\right)$ is estimated to be $-20 \%$ and $+15 \%$;

of Long distance $\mathrm{QCD}$ dynamics is described by the matrix elements, which are parametrized as

$$
\begin{aligned}
\left\langle\bar{B}_{s}\left|O_{1}(\mu)\right| B_{s}\right\rangle & \equiv\left\langle\bar{B}_{s}\left|(\bar{b} s)_{V-A}(\bar{b} s)_{V-A}\right| B_{s}\right\rangle=\frac{8}{3} f_{B_{s}}^{2} m_{B_{s}}^{2} B_{1}(\mu) \\
\left\langle\bar{B}_{s}\left|O_{2}(\mu)\right| B_{s}\right\rangle & \equiv\left\langle\bar{B}_{s}\left|(\bar{b} s)_{S-P}(\bar{b} s)_{S-P}\right| B_{s}\right\rangle=-\frac{5}{3}\left(\frac{f_{B_{s}} m_{B_{s}}^{2}}{m_{b}(\mu)+m_{s}(\mu)}\right)^{2} B_{2}(\mu) .
\end{aligned}
$$

The above parameters $B_{1,2}$ are equal to unity in the vacuum saturation approximation (VSA). A priori, VSA gives a gross estimate and one has to include the (non-factorizable) non-perturbative QCD effects. QCD simulations on the lattice represent a suitable method for that part of the job.

This year progress in reducing uncertainties of the heavy quark extrapolation of the $B$ -

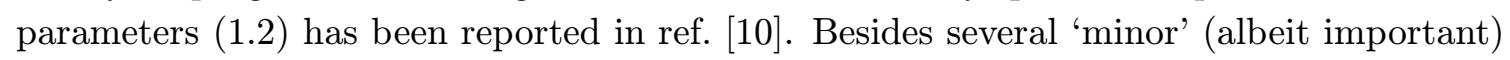
improvements, we combined the static HQET results of ref. [i, with those of ref. [igi], where lattice QCD is employed for the mesons of masses, $1.8 \mathrm{GeV} \lesssim m_{P} \lesssim 2.4 \mathrm{GeV}$. To use the HQ scaling laws we matched the QCD matrix elements with the HQET ones, so that we could actually "interpolate" to the mass of the $B_{s}$-meson. The obtained results are then matched back to their full QCD values. This matching HQET $\leftrightarrow$ QCD $\left(C_{B}\left(m_{P}\right)\right.$ in fig. ??) is for the first time made at NLO in perturbation theory. For consistency, the computation of the $B$-parameters is performed precisely in the $\overline{\mathrm{MS}}(\mathrm{NDR})$ scheme in which the functions $G_{i}(\mu)$ have been calculated [i]

$$
B_{1}\left(m_{b}\right)=0.87(2)(5), \quad B_{2}\left(m_{b}\right)=0.84(2)(4),
$$

${ }^{*} \mathrm{SV}$ (Shifman-Voloshin limit) is the limit in which $\Lambda_{\mathrm{QCD}} \ll m_{b}-2 m_{c} \ll m_{b}\left[{ }_{5}\right]$ ]. 
where the first errors are statistical and the second include various sources of systematics. The above results are obtained in the quenched approximation $\left(n_{f}=0\right)$, and the systematic error due to quenching could not be estimated. This year's novelty is the research made in that direction by the JLQCD collaboration [1 $1 \overline{1}_{1}^{1}$. Within the NRQCD approach, they examined the effect of inclusion of the dynamical quarks. They conclude that the $B$ parameters are essentially insensitive to switching from $n_{f}=0$ to $n_{f}=2$. From their (high statistics) unquenched simulation, they quote

$$
B_{1}\left(m_{b}\right)=0.87(4)(7), \quad B_{2}\left(m_{b}\right)=0.86(3)(7) .
$$

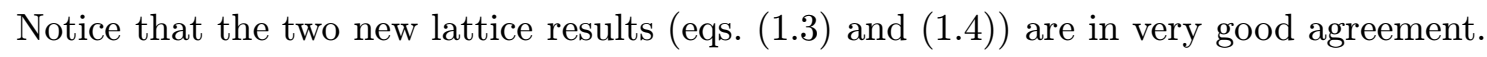

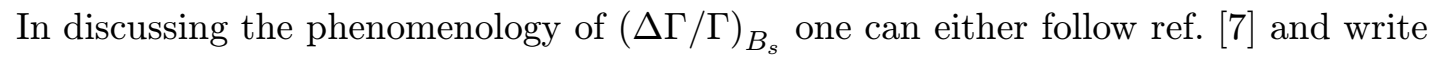

$$
\left(\frac{\Delta \Gamma}{\Gamma}\right)_{B_{s}}=\left(5.03(32) \mathrm{GeV}^{-2}\right) \cdot f_{B_{s}}^{2} B_{1}\left(m_{b}\right) \mathcal{M}\left(m_{b}\right)
$$

where $\mathcal{M}\left(m_{b}\right)=G_{1}\left(m_{b}\right)-G_{2}\left(m_{b}\right) \frac{\left\langle\bar{B}_{s}\left|O_{2}\left(m_{b}\right)\right| B_{s}\right\rangle}{\left\langle\bar{B}_{s}\left|O_{1}\left(m_{b}\right)\right| B_{s}\right\rangle}+\delta_{1 / m}$,

or, as proposed in [iפ], to write

$$
\left(\frac{\Delta \Gamma}{\Gamma}\right)_{B_{s}}=\left(1.09(10) \times 10^{-5}\right) \cdot \frac{\left(\tau_{B_{s}} \Delta m_{d}\right)^{\mathrm{EXP}}}{\left|V_{t b} V_{t d}\right|^{2}} \xi^{2} \mathcal{M}\left(m_{b}\right) .
$$

Indeed, by using $\xi^{2}$ and the experimental value for $\Delta m_{d}=0.519(20)(16) \mathrm{ps}^{-1}$ [1른 avoid the multiplication by $f_{B_{s}}$, for which the uncertainty is much larger than the one for the ratio $\xi=f_{B_{s}} \sqrt{B_{1}^{(s)}} / f_{B_{d}} \sqrt{B_{1}^{(d)}}=1.15(6)$ [1] $\underline{1}_{-1} \bar{j}$, where many of the systematic errors

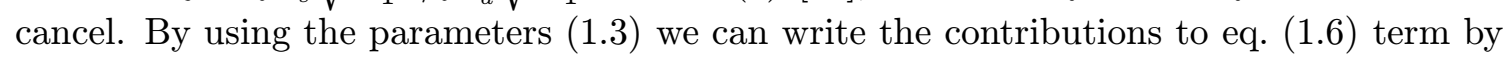
term

$$
\left(\frac{\Delta \Gamma}{\Gamma}\right)_{B_{s}}=0.005(9)+0.146(28)-0.086(19)=6.5 \pm 2.0_{-2.1}^{+1.3}=6.5_{-2.9}^{+2.4} \%,
$$

where I also used $\delta_{1 / m}=-0.5(1)$, as it can be obtained by applying the VSA to estimate the values of all the matrix elements that contribute at $1 / m_{b}$ (identified in ref. $[\bar{i} \mid \bar{b}]$ ). The last

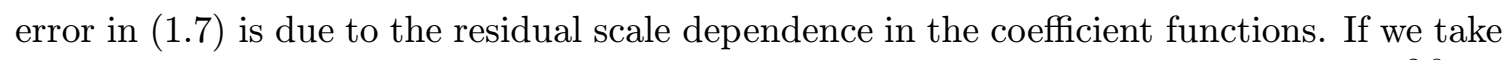

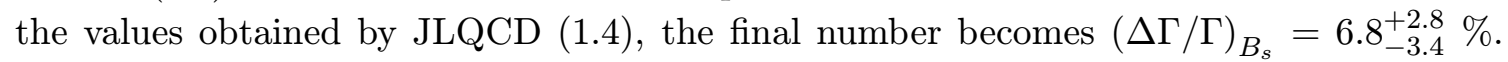
The final values are thus numerically small (much below the experimental limit). From eq. (1) $\left.\overline{1}_{1}\right)$ we also see why it is so: $1 / m_{b}$ corrections are very large and are of the sign opposite w.r.t. the second term, which would otherwise dominate eq. (1;.6in). The matrix elements that are present in $\delta_{1 / m}$ are very hard to compute and it will take quite some time before the lattice results for $\delta_{1 / m}$ appear. This is why it does not make sense to look for the physics beyond the SM from this quantity before taming the $1 / m_{b}$ corrections.

\section{RATIOS OF THE $B$-MESON LIFETIMES}

The spectator effects, responsible for the hierarchy of the heavy meson lifetimes, start showing up in OPE with the term $\propto 1 / m_{b}^{3}$. Out of many $\Delta B=0$ local operators contributing 
at that order, only a few are expected to be relevant to the ratios $\tau\left(B_{u(s)}\right) / \tau\left(B_{d}\right)$. These have been identified in ref. [i] $[1]-1]$, and parametrized as follows:

$$
\begin{aligned}
\left\langle B_{q}\left|(\bar{b} q)_{V-A}(\bar{q} b)_{V-A}\right| B_{q}\right\rangle & =f_{B_{q}}^{2} m_{B_{q}}^{2} B_{1}(\mu) \quad, \quad\left\langle B_{q}\left|\left(\bar{b} \frac{\lambda^{i}}{2} q\right)_{V-A}\left(\bar{q} \frac{\lambda^{i}}{2} b\right)_{V-A}\right| B_{q}\right\rangle=f_{B_{q}}^{2} m_{B_{q}}^{2} \varepsilon_{1}(\mu), \\
\left\langle B_{q}\left|(\bar{b} q)_{S-P}(\bar{q} b)_{S+P}\right| B_{q}\right\rangle & =f_{B_{q}}^{2} m_{B_{q}}^{2} B_{2}(\mu) \quad, \quad\left\langle B_{q}\left|\left(\bar{b} \frac{\lambda^{i}}{2} q\right)_{S-P}\left(\bar{q} \frac{\lambda^{i}}{2} b\right)_{S+P}\right| B_{q}\right\rangle=f_{B_{q}}^{2} m_{B_{q}}^{2} \varepsilon_{2}(\mu) .
\end{aligned}
$$

In the VSA, the above parameters are expected to be $B_{1}^{\mathrm{VSA}}=1, B_{2}^{\mathrm{VSA}}=\left[m_{B_{q}} /\left(m_{b}+\right.\right.$ $\left.\left.m_{q}\right)\right]^{2} \approx 1.5$, and $\varepsilon_{1,2}^{\mathrm{VSA}}=0$. The final expression for $\tau\left(B_{u}\right) / \tau\left(B_{d}\right)$ can be written as

$$
\begin{aligned}
\frac{\tau\left(B^{+}\right)}{\tau\left(B^{0}\right)}=1+16 \pi^{2} \frac{f_{B}^{2} m_{B}}{m_{b}^{3} c_{3}\left(m_{b}\right)} & \left\{G_{1}^{s s}\left(m_{b}\right) B_{1}\left(m_{b}\right)+G_{1}^{o o}\left(m_{b}\right) \varepsilon_{1}\left(m_{b}\right)\right. \\
+ & \left.G_{2}^{s s}\left(m_{b}\right) B_{2}\left(m_{b}\right)+G_{2}^{o o}\left(m_{b}\right) \varepsilon_{2}\left(m_{b}\right)+\bar{\delta}_{1 / m_{b}}\right\} .
\end{aligned}
$$

The main ingredients in this formula are:

$\circledast 16 \pi^{2}$ is the ("famous") phase space enhancement of the corrections $\left(\propto 1 / m_{b}^{3}\right)$;

$\circledast c_{3}\left(m_{b}\right)$ is the coefficient of the leading order term $\left(\propto 1 / m_{b}^{0}\right)$ which can be obtained by using the measured $b$-quark semileptonic branching fraction $B_{S L}^{\mathrm{EXP}}=\Gamma(B \rightarrow$ $X e \nu) / \Gamma_{T O T}=10.6(3) \%$ [1] $\left.{ }_{1}\right]$, and combine it with the theoretical expression for $\Gamma(B \rightarrow X e \nu)$ [1] $\left.{ }_{1}^{1} \overline{\sigma_{1}}\right]$. I obtain, $c_{3}\left(m_{b}\right)=3.8(1)(3)$;

$\circledast G_{1,2}(\mu)$ are the functions describing the short distance QCD dynamics of $\Delta B=0$ operators. This year these functions have been computed at the NLO accuracy. The results are:

$$
\begin{aligned}
& G_{1}^{s s}\left(m_{b}\right)=0.55, G_{1}^{s s}\left(m_{b}\right)=8.08 \\
& G_{1}^{s s}\left(m_{b}\right)=0.03, G_{1}^{s s}\left(m_{b}\right)=1.80
\end{aligned}
$$

$\circledast \bar{\delta}_{1 / m_{b}}$ stands for the neglected terms $\propto 1 / m_{b}^{3}$ which are not enhanced by " $16 \pi^{2}$ ", and for the terms in OPE that are $\propto 1 / m_{b}^{4}$. No research in that direction has been made.

\begin{tabular}{|c|c|c|}
\hline Sum rules (HQET) $\left[\begin{array}{l}{[2 \overline{0}} \\
2\end{array}\right]$ & Lattice HQET [1] & $\underline{\text { Lattice QCD }[\overline{1} \overline{1} \overline{\underline{9}}]}$ \\
\hline$B_{1}\left(m_{b}\right)=1.01(1)$ & $B_{1}\left(m_{b}\right)=1.06(8)$ & $B_{1}\left(m_{b}\right)=1.10(13)\left(\begin{array}{l}+.10 \\
-.21\end{array}\right)$ \\
\hline$B_{2}\left(m_{b}\right)=0.99(1)$ & $B_{2}\left(m_{b}\right)=1.01(6)$ & $B_{2}\left(m_{b}\right)=0.79(5)(9)$ \\
\hline$\varepsilon_{1}\left(m_{b}\right)=-0.08(2)$ & $\varepsilon_{1}\left(m_{b}\right)=-0.01(3)$ & $\varepsilon_{1}\left(m_{b}\right)=-0.02(2)\left(\begin{array}{l}+.01 \\
-.00\end{array}\right)$ \\
\hline$\varepsilon_{2}\left(m_{b}\right)=-0.01(3)$ & $\varepsilon_{2}\left(m_{b}\right)=-0.01(2)$ & $\varepsilon_{2}\left(m_{b}\right)=0.03(1)\left(\begin{array}{l}+.01 \\
-.00\end{array}\right)$ \\
\hline
\end{tabular}

$\circledast$ Until this year, there was only one lattice study of the matrix elements $\left(\overline{2} \cdot \overline{2}_{-} \overline{1}_{1}\right)$, and that one was made in the static limit of HQET [1] $\left.\overline{1}_{-1}\right]$. This year, the first lattice QCD computation of $\Delta B=0$ operators has been performed [i] $\bar{g}_{-1}^{1}$. Besides lattice simulations, also the QCD sum rule result was produced in ref. [i2 $\overline{2} \overline{0}]$. The compendium of the present results looks as follows: 
After combining all of the above results and by using the new bag parameters obtained in QCD, as well as $f_{B}=0.198(30) \mathrm{GeV}$ [1]

$$
\frac{\tau\left(B^{+}\right)}{\tau\left(B^{0}\right)}=1.067(27) \simeq 1.07(3) .
$$

It is important to note that in the lattice computation of the bag parameters no penguinlike contractions are accounted for. The justification for that ommission was provided in ref. [īind. That also means that the present results for bag parameters cannot be used to estimate the difference $B_{1}(\mu)-B_{2}(\mu)$, which is relevant for the precision extraction of $\left|V_{u b}\right|$ from the inclusive semileptonic $B \rightarrow X_{u} \ell \nu_{\ell}$ decay [2] $\left.{ }_{-}^{1}\right]$.

\section{Summary and prospects}

After several years of exciting research to reduce the theoretical uncertainty on $(\Delta \Gamma / \Gamma)_{B_{s}}$, the leading term in OPE for this quantity is in good shape: NLO perturbative corrections and quite reliable estimates of the matrix elements, obtained from new lattice studies, are available. However, a rough estimate of the subleading $\left(1 / m_{b}\right)$ corrections in the OPE indicates that such corrections, to a large extent, wash out the effect of the leading terms: the corrections are large and of opposite sign. Therefore, as of now, it does not seem reasonable to test the Standard Model (or to expect to see the signal of physics beyond the Standard Model) from this quantity. It is, in fact, necessary to improve the theoretical predictions by taming the dimension-seven operators (the ones that enter with $1 / m_{b}$ corrections). From the present theoretical situation I conclude that

$$
\left(\frac{\Delta \Gamma}{\Gamma}\right)_{B_{s}}=(7 \pm 4) \%
$$

This year, a further theoretical improvement in the lifetime ratios of the $B$-mesons has been made. QCD radiative corrections to the coefficient functions are now being calculated, and the first QCD computation of the bag parameters performed (which is complementary to the earlier lattice HQET results). Lattice predictions will certainly improve in many respects (e.g. non-perturbative renormalization will be carried out, the penguin-like contractions are likely to be included in the HQET lattice studies, unquenched simulations in HQET will become feasible,...) Note that the operators that give rise to $1 / m_{b}$ corrections to the non-spectator effects in $\tau\left(B_{u, s}\right) / \tau\left(B_{d}\right)$ are yet to be identified and their effects estimated. Such a study would be very welcome. From the present theoretical information, for the ratios of the $B$-meson lifetimes I obtain

$$
\frac{\tau\left(B^{+}\right)}{\tau\left(B^{0}\right)}=1.07(3), \quad \frac{\tau\left(B_{s}\right)}{\tau\left(B_{d}\right)}=1.00(2) .
$$

\section{Acknowledgement}

It is a pleasure to thank my friends and collaborators of refs. [i9i], [i $\left.{ }_{1}^{1}\right]$ and also those of ref. [i] $\left.\overline{1}_{1}\right]$, for sharing their insights in topics covered by this talk. Communication with S. Ryan, N. Yamada and the authors of ref. [1] $1 \overline{1}$ 8i] is kindly acknowledged. 


\section{References}

[1] D. Abbaneo et al. [LEP, SLD, CDF], hep-ex/0009052; D. Abbaneo, hep-ex/0108030.

[2] M. A. Shifman, hep-ph/0009131.

[3] A. Pich, Nucl. Phys. Proc. Suppl. 98 (2001) 385, hep-ph/0012297.

[4] R. Aleksan, A. Le Yaouanc, L. Oliver, O. Pene and J. C. Raynal, Phys. Lett. B 316 (1993) 567.

[5] M. A. Shifman and M. B. Voloshin, Sov. J. Nucl. Phys. 47 (1988) 511.

[6] M. Beneke, G. Buchalla and I. Dunietz, Phys. Rev. D 54 (1996) 4419, hep-ph/9605259.

[7] M. Beneke, G. Buchalla, C. Greub, A. Lenz and U. Nierste, Phys. Lett. B 459 (1999) 631, hep-ph/9808385.

[8] V. Gimenez and J. Reyes, Nucl. Phys. Proc. Suppl. 93 (2001) 95, hep-lat/0009007.

[9] D. Becirevic, D. Meloni, A. Retico, V. Gimenez, V. Lubicz and G. Martinelli, Eur. Phys. J. C 18 (2000) 157, hep-ph/0006135;

D. Becirevic, Nucl. Phys. Proc. Suppl. 94 (2001) 337, hep-lat/0011075.

[10] D. Becirevic, V. Gimenez, G. Martinelli, M. Papinutto and J. Reyes, hep-lat/0110091.

[11] S. Hashimoto and N. Yamada [JLQCD collaboration], hep-ph/0104080; N. Yamada et al. [JLQCD Collaboration], hep-lat/0110087.

[12] B. Aubert et al. [BABAR Collaboration], hep-ex/0107036.

[13] C. W. Bernard, Nucl. Phys. Proc. Suppl. 94 (2001) 159, hep-lat/0011064;

S. Ryan, hep-lat/0111010.

[14] M. Neubert and C. T. Sachrajda, Nucl. Phys. B 483 (1997) 339, hep-ph/9603202.

[15] C. S. Lin, hep-ex/0105025.

[16] Y. Nir, Phys. Lett. B 221 (1989) 184.

[17] M. Ciuchini, E. Franco, V. Lubicz and F. Mescia, hep-ph/0110375.

[18] M. Di Pierro and C. T. Sachrajda, Nucl. Phys. B 534 (1998) 373, hep-lat/9805028.

[19] D. Becirevic et al., "B-meson lifetime ratios: Lattice $Q C D$ estimate of $\Delta B=0$ operators", in preparation.

[20] M. S. Baek, J. Lee, C. Liu and H. S. Song, Phys. Rev. D 57 (1998) 4091, hep-ph/9709386.

[21] M. B. Voloshin, Phys. Lett. B 515 (2001) 74, hep-ph/0106040. 